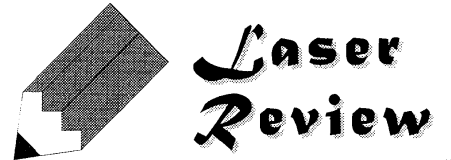

\section{Na Laser Guide Star Technologies for Adaptive Imaging}

\author{
Chester S. GARDNER \\ Office of the Vice President, University of Illinois \\ 377 Henry Administration Building, 506 South Wright Street, Urbana, IL 61801
}

(Received October 13, 2000)

\begin{abstract}
Atmospheric turbulence severely limits the resolution of groundbased astronomical telescopes. In good seeing conditions at the best observatory sites, resolution at visible wavelengths is typically limited to - $1 \mathrm{~s}$ of arc. During the past 20 years adaptive optical systems using electrically deformable mirrors have been developed to compensate for turbulence effects. Unfortunately, these systems require bright reference sources adjacent to the object of interest and can be used only to observe the brightest stars. Artificial guide stars suitable for controlling an adaptive imaging system can be created in the upper atmosphere by using a laser to excite resonance backscattering in the mesospheric sodium layer. The design requirements of a laser-guided adaptive telescope, as well as the expected imaging performance, are reviewed in this paper.
\end{abstract}

Key Words: Laser guide star, Adaptive optics, Diffraction limited imaging, Na layer, Atmospheric turbulence

\section{Introduction}

The resolution of large ground-based telescopes is limited by random wavefront distortion caused by atmospheric turbulence. However, with the use of adaptive optics techniques these telescopes can achieve near diffraction-limited imaging performance. The operation of an adaptive optics system consists of two basic functions: wavefront sensing and wavefront correction. By using the light from either the object being observed or a nearby guide star, a wavefront sensor measures the turbulence induced phase perturbations across the telescope aperture. The measurements, in turn, are used to adjust an electrically deformable mirror to compensate for the phase distortions. This general class of the adaptive optics system has been shown to work if 1) the reference wave front from the guide star is within the isoplanatic angle of the object being observed and 2) if the guide star is bright enough to provide an adequate signal for the wavefront sensor. For astronomical imaging, these two requirements imply that bright guide stars must be available within the isoplanatic angle for all observation directions. Because the density of bright stars is low, an adaptive telescope based on natural guide stars does not provide adequate sky coverage for astronomical imaging at visible wavelengths.

Foy and Labeyrie ${ }^{1)}$ in 1985 suggested that it may be possible to create a suitable artificial guide star in the upper atmosphere with a laser. Laser guide stars would allow access to nearly the entire sky even at visible wavelengths since the laser could be pointed in any desired direction. In 1987 Thompson and Gardner ${ }^{2)}$ further advanced the laser guide star concept by demonstrating experimentally the feasibility of creating a laser guide star in the mesospheric Na layer. In the early 1990s as civilian researchers began identifying the key engineering challenges and demonstrating the potential imaging capabilities, ${ }^{3)}$ the US military declassified much of its extensive work on adaptive imaging and laser guide stars. Since then numerous research papers have appeared in the scientific literature which have stimulated further work in this important field. ${ }^{4)}$ Consequently, during the past decade, adaptive imaging technology has found widespread use in major astronomy facilities worldwide. ${ }^{5)}$

This paper is a condenced updated summary of the work published by Gardner et al. ${ }^{3)}$ We summarize the basic concepts and engineering issues important for the design of laser guided adaptive telescope systems. We consider laser guide stars created using resonance-fluorescence scattering at a relatively high altitude within the mesospheric sodium ( $\mathrm{Na}$ ) layer. Computing the rcquired brightness of the guide star is one of the major design tasks. Other design issues include the method of forming the guide star, the positional stability of the guide star, the required size of the guide star, and the number of guide stars needed for a given telescope diameter.

\section{Laser Guided Adaptive Telescope Design}

Consider first a small diameter telescope. A diffraction limited image is focused onto the image plane of the telescope if the pupil diameter $D$ is less than the Fried ${ }^{6)}$ coherence cell size $r_{\mathrm{o}}$. The angular resolution of the telescope in the short exposure limit is $1.22 \lambda D$, where $\lambda$ is the optical wavelength. However, for long exposures, random wavefront tilts cause the image to dance around in the focal plane and image blurring occurs. If the object is bright enough, a simple tip-tilt mirror arrangement can be used to compensate for the random wavefront tilt. Such a system would consist of three main components: an image centroid detector, a tip-tilt mirror, and a control algorithm connecting the two.

For a telescope diameter larger than $r_{\mathrm{o}}$, the angular resolution is limited to $1.22 \lambda / r_{\mathrm{o}}$. Each subaperture of size $r_{\mathrm{o}}$ within the telescope pupil generates a subimage of angular radius 1.22 $\lambda / r_{0}$. A random wavefront tilt over each $r_{\mathrm{o}}$ subaperture causes the subimages to dance around in the image plane. The motion 
of a single subimage is nearly independent of the motion of every other subimage. Modern adaptive optics telescopes use a wavefront sensor to measure the wavefront tilts over each $r_{\mathrm{o}}$ subaperture. One of the more common wavefront sensors is the Hartmann sensor which consists of a lenslet array and detector array. The detector array measures the centroid displacement of the image spot under each lenslet. These measurements are in turn used to reconstruct an estimate of the wavefront phase surface, which is then used to control the shape of a deformable mirror. The wavefront phase perturbations are nearly canceled and the telescope approaches diffraction limited performance.

The major components of a laser-guided adaptive telescope (Fig.1) include the laser, laser beam jitter sensor, low-frequency motion sensor, deformable mirror, tip-tilt mirror, wavefront sensor, imaging module, and control module. ${ }^{3,4)}$ The laser beam is expanded and transmitted through a projection telescope behind the Cassegrain secondary mirror. The beam is focused at the altitude of the selected atmospheric scattering region. Before leaving the projection telescope, part of the beam is sampled for use by the jitter sensor. The jitter sensor measures the propagation angle of the laser beam as it exits the laser, and this information is fed back into the control module to null out effects of laser induced beam jitter. Figure 2 is a photograph of the University of Illinois Na laser beam and $3.5 \mathrm{~m}$ telescope operated by the Air Force Research Laboratory, Directed Energy Directorate at the Starfire Optical Range near Albuquerque, NM.

The back scattered light from the laser guide star, after being focused by the telescope, is passed through the central hole of a turning mirror. The mirror reflects the light from a set of natural stars within the field of view of the telescope. The low-frequency motion sensor locks onto the location of these stars and sends a signal to the control module where the low-frequency motions of the laser guide star and telescope are nulled. After passing through the turning mirror the light from the laser guide star is collimated and is reflected first off the tip-tilt mirror and then off the deformable mirror. The tip-tilt mirror removes overall wavefront tilt while the deformable mirror is adjusted to mini-

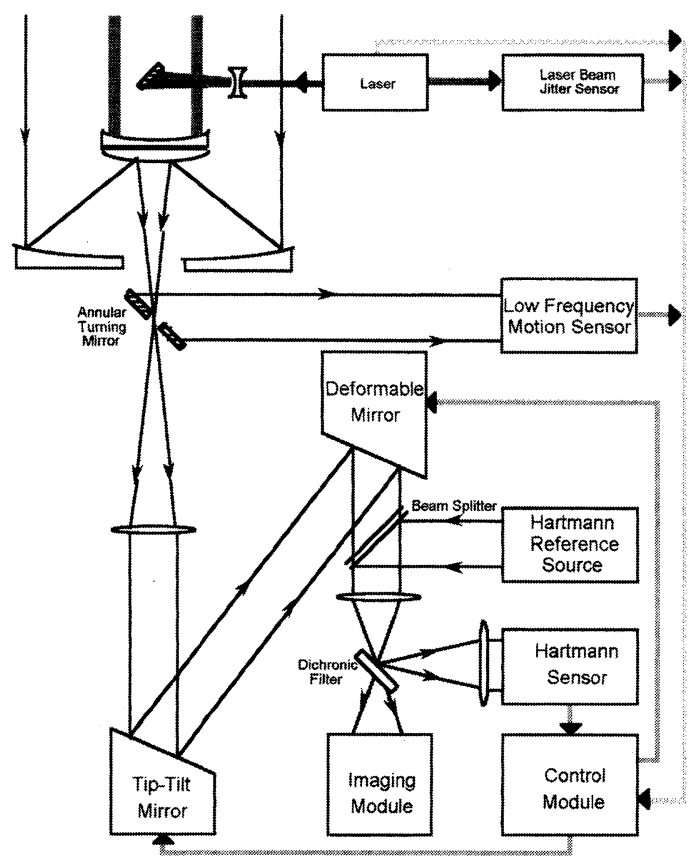

Fig.1 Schematic diagram of a laser guided adaptive telescope.

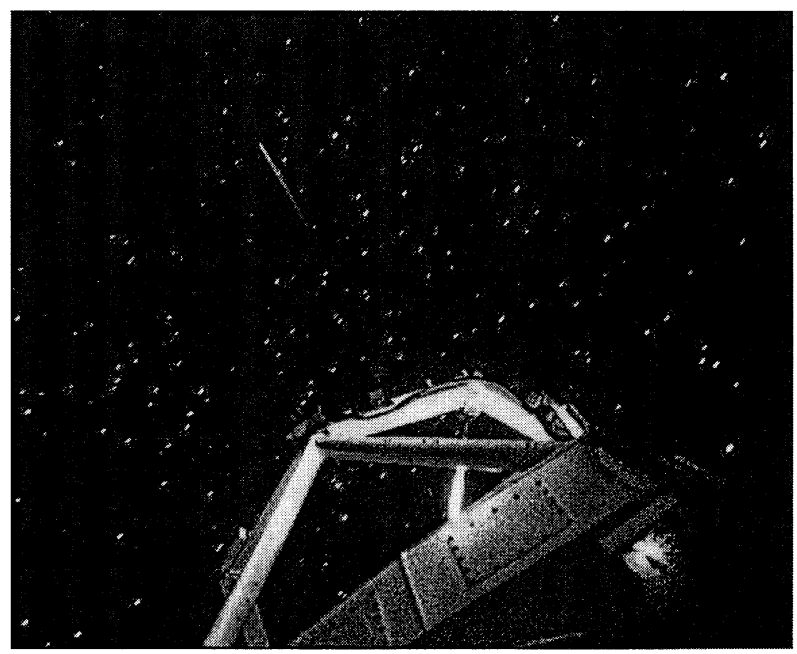

Fig.2 Na laser beam projected from the $3.5 \mathrm{~m}$ telescope at the Starfire Optical Range near Albuquerque, New Mexico.

mize the residual phase errors. The light is then reflected off a dichroic beam splitter which transmits the Na light $(589 \mathrm{~nm})$ to the wavefront sensor. The remaining light is directed to the imaging module. The subaperture tilts detected by the wavefront sensor are processed and sent to the deformable mirror as actuator control signals. The imaging module captures the corrected image reflected from the deformable mirror and these data are saved as the final astronomical image.

In an ideal system the wavefront compensation would result in perfect cancellation of the phase perturbations of the aberrated wave front. In reality, though, there are many factors which prevent perfect cancellation. Two factors are our inability to build perfect wavefront sensors and deformable mirrors. Hardy ${ }^{7)}$ presents a thorough review of the types of wavefront sensors and deformable mirrors used in modern adaptive optics. The two most important limiting factors are 1) the spacing of the sensors and mirror actuators within the aperture of the optical system and 2) photon noise. Sensor and actuator spacing determines the response of the adaptive optics system to the highest spatial frequency of the phase perturbations. From the discussion above we indicate that tilt sensors and tilt correctors spaced at intervals of $r_{\mathrm{o}}$ can result in nearly diffraction limited imaging performance. Even with this spacing the accuracy of the reconstructed wave can be severely degraded if photon noise effects are significant. Photon noise arises because of the finite flux available from the guide star reference wavefront. A weak reference wavefront can significantly degrade performance. Quantifying the effects of photon noise is particularly important for the design of laser-guided adaptive telescopes since the laser requirements are derived directly from the guide star brightness requirement.

\section{Atmospheric Turbulence Effects}

During the past 15 years many authors have studied the effects of atmospheric turbulence on ground-based imaging systems. ${ }^{8-11)}$ In 1966 Fried $^{8)}$ wrote a seminal paper describing the performance of an optical system in the presence of atmospheric turbulence. He characterized performance in terms of the OTF. From the OTF Fried derived a single parameter, now called the Fried coherence diameter $r_{0}$, that concisely characterizes the per- 
formance of an aperture in the presence of atmospheric turbulence. By using a measure of resolution equivalent to the Strehl ratio, Fried showed that for a telescope diameter $D$ much less than $r_{\mathrm{o}}$, resolution is proportional to the square of $D$. As $D$ becomes larger than $r_{\mathrm{o}}$, resolution asymptotically approaches a constant value exactly equal to the theoretical resolution of an aperture of diameter $r_{\mathrm{o}}$. The dependence of $r_{\mathrm{o}}$ on wavelength goes as $\lambda^{6 / 5}$. As the wavelength increases the effect of atmospheric turbulence decreases. At the best observatory sites, and in conditions of good seeing, $r_{\mathrm{o}}$ can range from 10 to $30 \mathrm{~cm}$ for visible wavelengths.

The dominant effect of atmospheric turbulence over an aperture of diameter $r_{\mathrm{o}}$ is a random wavefront tilt. This assertion was quantified by Fried ${ }^{11)}$ who showed that for an adaptive optics system capable of achieving perfect tilt compensation, the average rms value of the wavefront error $\Delta \phi$ (difference between the actual and tilt compensated wavefront expressed as a path length error in units of wavelengths) over an aperture of diameter $d$ is given by

$$
\Delta \phi=\left(\frac{d}{r_{0}}\right)^{5 / 6} \frac{\lambda}{17.4}
$$

Equation (1) indicates that a large part of the phase distortions across an aperture can be attributed to wavefront tilt. In fact nearly $90 \%$ of the power in the phase fluctuations are due to tilt. This is an important result since it indicates that an adaptive optics system correcting for wavefront tilt can remove a large percentage of the phase distortions. The smaller the aperture over which the tilt is removed the smaller the residual uncorrected phase variation.

Consider an aperture segmented into subapertures of diameter $r_{\mathrm{o}}$. If the overall aperture and subaperture tilts are removed, according to (1) the resulting rms wavefront error over the entire aperture will be $\lambda / 17.4$. This wavefront phase variation yields a Strehl ratio of 0.88 . By comparison, the wavefront error (small scale roughness) for the Hubble Space Telescope primary and secondary mirror has been measured to be $\lambda / 21.4$ for a wavelength of $632.8 \mathrm{~nm}$, which corresponds to a theoretical Strehl ratio of 0.92 . This simple example demonstrates that it is possible to achieve near diffraction limited performance simply by removing wavefront tilt over subapertures of diameter $r_{\mathrm{o}}$.

\section{Na Laser Guide Stars}

The brightness of the guide star determines the ultimate accuracy of the wavefront sensor and consequently the performance of the adaptive telescope. The brightness requirement and the associated power requirements of the laser used to form the $\mathrm{Na}$ guide star have a direct impact on the performance of the adaptive telescope. This complex but important issue is addressed in detail elsewhere ${ }^{3,4)}$ and will not be repeated here.

The mesospheric $\mathrm{Na}$ layer provides an excellent scattering medium for creation of laser guide stars because of its high altitude and its large resonant backscattering coefficient. The layer was discovered in the late-1920's and has been explored extensively since the late-1960's with lidar techniques. The Na layer is created by meteoric ablation, and depleted at its lower boundary by chemical processes. The layer lies at a mean altitude of $92 \mathrm{~km}$, and its average thickness is about $10 \mathrm{~km}$ FWHM. The Na column density varies on both diurnal and annual time scales. The column abundance at mid-latitudes in the Northern Hemisphere shows a summer minimum of about $2.5 \times 10^{9} \mathrm{~cm}^{-2}$ and a winter maximum of about $5 \times 10^{9} \mathrm{~cm}^{-2}$ in November through January. ${ }^{12)}$ The seasonal and geographical variations are believed to be related to changes in mesopause temperatures that affect the main chemical reaction rates at the lower boundary of the Na layer. Diurnal variations in the $\mathrm{Na}$ abundance are caused primarily by the influence of atmospheric tides, gravity waves, and solar radiation. The abundance is minimum a few hours before local midnight and maximum just before sunrise. Figures 3 and 4 illustrate the seasonal and diurnal variations of the Na layer column abundance and centroid height as measured at Urbana, Illinois. Figure 5 is a Na density profile measured during the 1998 Leonid meteor shower at the Starfire Optical Range, NM. The thin dense layers near 92 and $98 \mathrm{~km}$ altitude are meteor ablation trails. ${ }^{13)}$

The process of selecting the laser pulse energy, pulse length, and linewidth to achieve the guide star brightness requirements is not straight forward because absorption by the $\mathrm{Na}$ layer is

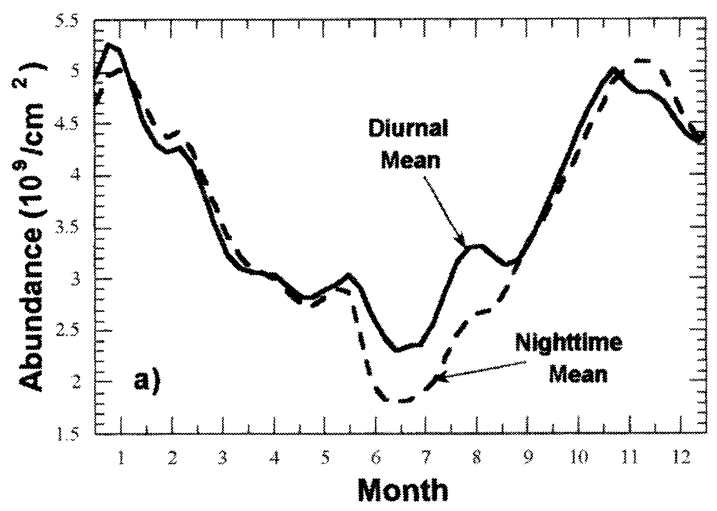

Fig.3 Seasonal variation of the Na layer abundance at Urbana, IL $\left(40^{\circ} \mathrm{N}\right)$. The diurnal mean was computed by averaging the data over $24 \mathrm{~h}$. The nighttime mean was computed using a weighted average of the nighttime data. ${ }^{12)}$
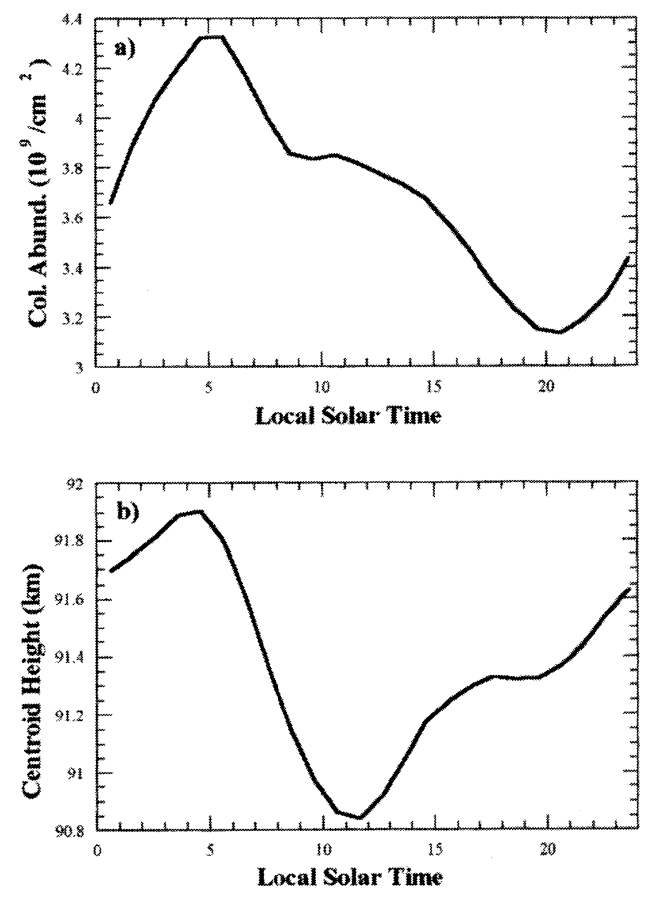

Fig.4 Diurnal variations of the a) Na layer abundance and b) centroid height at Urbana, IL $\left(40^{\circ} \mathrm{N}\right) .{ }^{12)}$ 


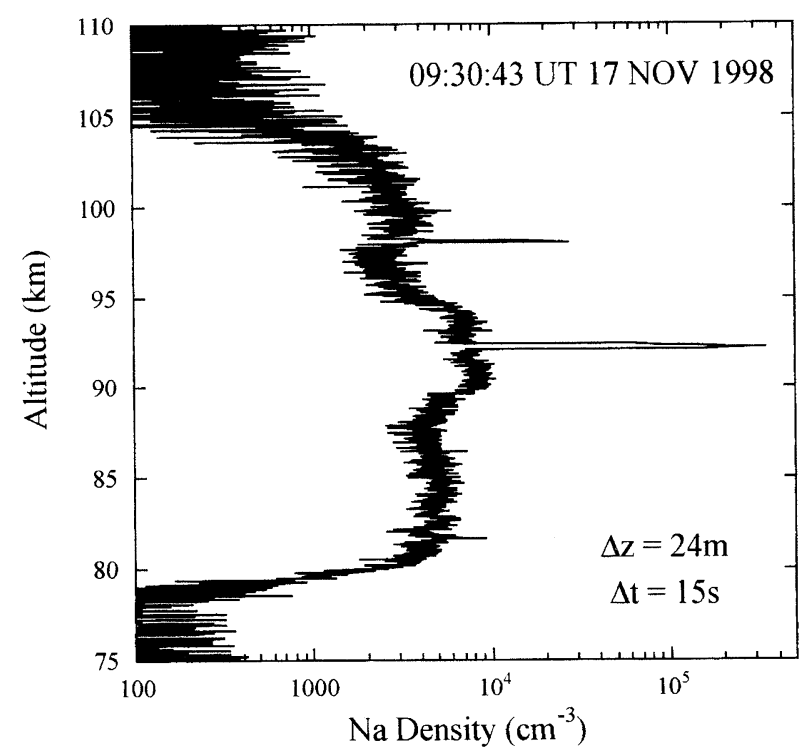

Fig.5 Na meteor trail profile probed by the Na wind/temperature lidar at the Starfire Optical Range during 1998 Leonid meteor shower. The meteor trails are located at 92 and $98 \mathrm{~km}$. The vertical resolution is $24 \mathrm{~m}$ and the integration period is $15 \mathrm{~s}$.

nonlinear. ${ }^{14)}$ Saturation arises when the energy density within the Na layer is large enough to significantly alter the population densities of the atomic states. In terms of a simple two-state system, a large energy density can significantly reduce the number of lower state atoms while increasing the number of excited state atoms. These altered state populations lead to nonlinear absorption of the laser energy, which results in an increased rate of stimulated emission and a reduced rate of fluorescence. The combined effect is a reduction in the backscattered energy from the guide star. To achieve the required guide star brightness, the laser pulse energy must be relatively large. This large pulse energy in combination with the small size of the guide star may contribute to significant saturation effects unless the laser parameters are chosen carefully. Fortunately, the laser design can be optimized in terms of the pulse energy, pulse length, and linewidth. ${ }^{14)}$

\section{Conclusions}

Ground-based adaptive telescopes using laser guide stars can produce images that are nearly diffraction limited. ${ }^{4,5)}$ The major challenge today is building lasers with the required characteristics. One of the more important design issues is determining the required laser power. The viability of building laser guided telescopes depends on the ability of the designer to minimize the laser power requirements in order to achieve power levels within range of today's laser technology. The main factors affecting the power requirements include the expected imaging performance $(\Delta \phi)$, the subaperture diameter $(d)$, and seeing conditions $\left(r_{0}\right)$. The required laser power is roughly proportional to $r_{\mathrm{o}}{ }^{-4}$ which illustrates the importance of choosing a site with extremely good seeing conditions (i.e., large $r_{\mathrm{o}} \sim 15-20$ $\mathrm{cm}$ ) in order to achieve reasonable laser power requirements. Although many technical challenges remain, the astronomy community has moved forward rapidly with implementing adaptive optics at its major facilities. As the technical problems of building and operating the guide star lasers are solved, the advantages of laser guided adaptive imaging and interferometry are certain to be quickly exploited in the next generation of optical astronomy facilities.

\section{References}

1) R. Foy and A. Labeyrie: Ast. Astrophys. 152 (1985) 129.

2) L. A. Thompson and C. S. Gardner: Nature 328 (1987) 229.

3) C. S. Gardner, B. M. Welsh, and L. A. Thompson: Proc. IEEE 11 (1990) 1721.

4) Atmospheric-Compensation Technology: J. Opt. Soc. Am. A 11 (1994) $257,783$.

5) R. Angel and R. Fugate: Science 288 (2000) 455.

6) D. L. Fried: J. Opt. Soc. Am. 56 (1966) 1372.

7) J. H. Hardy: Proc. IEEE 66 (1978) 651.

8) D. L. Fried: J. Opt. Soc. Am. 56 (1966) 1380.

9) H. T. Yura: Proc. SPIE 75 (1976) 9.

10) R. S. Lawrence: Proc. SPIE 75 (1976) 2.

11) D. L. Fried: J. Opt. Soc. Am. 55 (1965) 1427.

12) R. States and C. S. Gardner: J. Geophys. Res. 104 (1999) 11,783.

13) X. Chu, A. Liu, G. Pepen, C. S. Gardner, M. Kelley, J. Drummond, and R. Fugate: Geophys. Res. Lett. 27 (2000) 1815.

14) J. R. Morris: J. Opt. Soc. Am. A 11 (1994) 832. 